\title{
Session 8: Molecular biology - II
}

Thursday 9 October 2003. Moderators: Alain Vasserot and Dee Athwal

[15.20-16.20]

Antibody specificities selected from n-CoDeR antibody gene libraries: Design, characterization and application

Eskil Soderlind

BioInvent Therapeutic AB, SE-223 70 Lund, Sweden

Tel: +46+46 2868577; 2868550; Fax: +46+46

2110806; E-mail: es@bioinvent.com

URL: http://www.bioinvent.com/

BioInvent is an antibody company focusing on the development of Therapeutic Antibodies, either in collaborations or by ourselves. BioInvent's proprietary $\mathrm{n}-\mathrm{CoDeR}^{\mathrm{TM}}$ antibody libraries have been used in the discovery process to identify several panels of specific antibody fragments, against a variety of antigens. In summary, antibody fragments selected from the nCoDeR library are highly functional with excellent affinities and demonstrate good expression in our GMPmanufacturing system. They are now be explored in Therapeutic Antibody development programs.

The technology platform is BioInvent's proprietary $\mathrm{n}-\mathrm{CoDeR}^{\mathrm{TM}}$ antibody libraries, which are built by recombining in vivo formed complementarity determining regions (CDR:s) into one single master framework. Highly specific and functional human antibody fragments have been selected using the $n-C o D e R^{T M}$ libraries using an integrated automated antibody discovery platform. This platform allows large panels of reactive antibody fragments to be selected, and highly specific assays to be developed, to select individual highly specific antibody fragments.

After the selection of antibody fragments, these can then be rapidly moved into Antibody Development programs, for the construction of all human IgG molecule. The resulting intact antibody molecules have been successfully manufactured in house under GMP conditions. The IgG molecules from the n-CoDeR system have high affinities and are highly expressed in our in house GMP-manufacturing system.
BioInvent has a proprietary project portfolio utilizing our in-house capabilities in antibody generation and GMP production. The most recent project aims to develop antibody drugs against the HIV-TAT protein for treatment of HIV infection and for the treatment of arteriosclerosis.

The latest result utilizing the n-CoDeR platform will be presented and discussed.

\section{[16.20-16.50]}

Generation of a diverse panel of high affinity, fully human monoclonal antibodies using human PBL engrafted SCID mice

John S. Abrams

Xenerex Biosciences, San Diego, USA

Abstract not received.

[16.50-17.20]

Angiomics: Identification of tumor angiogenesis-associated antigens by means of antibody phage display

Yong-Jiang Cao ${ }^{\mathrm{a}}$, Jörg Willuda ${ }^{\mathrm{a}}$, Heike Petrul ${ }^{\mathrm{a}}$, Thomas Wäckerle ${ }^{\mathrm{a}}$, Stefan Rosewicz ${ }^{\mathrm{b}}$, Andreas Menrad $^{\mathrm{a}}$ and Klaus Bosslet ${ }^{\mathrm{a}}$

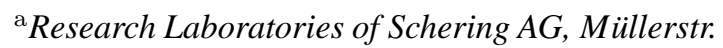
178, D-13342 Berlin, Germany

${ }^{\mathrm{b}}$ Medical Clinic for Hepatology/Gastroenterology, Charité, CVK, Augustenburgerplatz 1, 13353 Berlin, Germany

Objectives: Tumor angiogenesis is a complex process in which the phenotype of the proliferating endothelial cells is strongly controlled by the tumor's microenvironment. Once endothelial cells are isolated and grown under cell culture conditions they undergo dramatic phenotypic changes ("phenotypic drift"). To avoid or minimize such in vitro artifacts which may hamper the identification of cell surface structures relevant for the process of tumor angiogenesis we have 
adapted antibody phage display strategies accordingly. The results of two different selection procedures are presented.

Methods: We have performed antibody phage display by competitive cell panning and tissue panning. For cell panning, cultured tumor-derived endothelial cells were incubated with an antibody phage display library (diversity: $5.2 \times 10^{9}$ ) and tumor endothelial cell bound phages were isolated. To demonstrate specificity for tumor endothelial cells, the eluted phage-antibodies were screened by ELISA on untreated tumor-derived endothelial cells and irrelevant cells as a negative control. All ELISA positive antibodies were further characterized by immunohistochemistry on human tumor tissues and benign tissue. For tissue panning, single cell suspensions from human tumor tissues were prepared by mild enzymatic digestion. Phages bound to tumor endothelial cells were isolated from irrelevant contaminating cells by means of magnetic bead selection. Specificity of phages for tumor vasculature was demonstrated by immunohistochemistry on human tumor- vs. benign tissue.

Results: We have successfully established two different antibody phage display technologies. In comparison, direct tissue panning was more efficient than cell panning. Screening by immunohistochemistry showed that $26 \%$ of randomly picked phage clones from tissue panning specifically stained blood vessels. Among them $8.5 \%$ of randomly picked phage clones were specific for tumor vasculature. Although cell panning on tumor endothelial cells poduced more positive clones for tumor endothelial cells by ELISA screening (40\% of randomly picked phage clones), only $17.7 \%$ clones specifically labeled blood vessels as demonstrated by immunohistochemistry. Approximately 6\% of randomly picked phage clones specifically labeled tumor vasculature.

Conclusion: Tissue panning and cell panning strategies provide powerful tools for the enrichment of tumor-endothelial cell specific phage-antibodies and thus have a high potential for the identification of novel antigens associated with the process of tumorangiogenesis.

[17.20-17.50]

Antibody discovery using human Fab phage

display libraries

David R. Buckler

Dyax Corporation, Cambridge, MA, USA
Dyax Corp has developed an integrated phage display discovery and development platform for rapidly identifying antibodies with high affinity and specificity for clinical targets. Dyax's phage-display technology provides key opportunities not accessible in hybridoma-based screening, including discovery of antibodies to non-immunogenic, toxic, or immunosuppressive targets and targets with signficant homology in humans and mice. Phage display also allows direct control of target binding conditions, use of targets that may have limited stability in in-vivo conditions, and a shortened time-line to antibody discovery. Critical hurdles in establishing an efficient phage-antibody discovery platform include strategic creation of a human antibody library with high diversity, establishment of automated high-throughput screening systems, and managing DNA sequence information to characterized isolated binders. This presentation will discuss aspects of the Dyax technology platform that have driven successful antibody discovery for a range of challenging targets. Dyax's proprietary human antibody libraries include synthetic-derived diversity in CDRs and are constructed with $\mathrm{V}$-genes from human autoimmune lymphocytes (WO 01/79481). High-throughput screening, DNA sequencing, and data management through a central database allow efficient sequence analysis, grouping, and prioritization of candidates for reformatting to full-length antibodies. Finally, a number of follow-on assays have been established to assess direct binding activity and functional properties of identified antibodies. Case studies will be presented that demonstrate the utility of Dyax's antibody phage display platform for rapid discovery of antibodies specific for targets in different conformational or activation states that bind with picomolar to nanomolar affinity for target (before affinity maturation).

[17.50-18.10]

Title to be confirmed

David Light

Berlex Biosciences, Richmond, California, USA

Abstract not received 
[18.10-18.30]

Cloning and expression of functional single chain variable fragments ( $\mathrm{scFv}$ ) directed against PVY proteins: Molecular analysis and insitu recognition of target proteins

Souad Rouis ${ }^{\mathrm{a}, *}$, Pierre Lafaye ${ }^{\mathrm{c}}$, Radhia

Gargouri-Bouzid $^{\mathrm{a}}$ and Hammadi Ayadi ${ }^{\mathrm{b}}$

${ }^{\mathrm{a}}$ Centre de Biotechnologie, 3018 Sfax, Tunisia

${ }^{\mathrm{b}}$ Laboratoire de Génétique Moléculaire Humaine,

Faculté de Médecine, 3029 Sfax, Tunisia

E-mail: hammadi.ayadi@fmsf.rnu.tn

${ }^{\mathrm{c}}$ Unité de génétique et Biochimie de développement,

Institut Pasteur Paris, France

${ }^{*}$ E-mail: souad.rouis@cbs.rnrt.tn

Potato virus $\mathrm{Y}$ is the type member of potyvirus group. It infects solanaceous causing devastating damages on culture. Potyvirus strand genomic RNA encodes a polyprotein of about $350 \mathrm{kDa}$ that is autocatalytically cleaved generating the different PVY proteins. several genes encoding for structural and non structural proteins have been expressed in plants in order to attenuate plant infection by this virus. However, risks due to expression of viral proteins may limit a wide application of these strategies. Production of transgenic plants resistant to potyvirus $\mathrm{Y}$ by expression of monoclonal antibodies directed against virus proteins, mainly capsid, were proposed as an alternative approach.
We have previously cloned hybridoma secreting monoclonal antibodies directed against PVY structural and functional proteins and we have showed that the one directed against NIa protein was able to inhibit PVY polyprotein cleavage by blocking the activity of its target protein early in infection.

In this work we report the synthesis, the expression and the comparative study of $\mathrm{scFv}$ obtained from monoclonal anrtibodies directed respectively against a functional and a structural PVY proteins. Amplification of these fragments was performed by direct cloning of the assembled PCR product into TOPO vector followed by direct sequencing. Positive clones were then subcloned into PHEN vector and expressed in TG1 ${ }^{\mathrm{TR}}$ strain of E.Coli. The level of expression and the activity of the different $\mathrm{scFv}$ were verified by ELISA, Western Blot and immunohistochemistry.

Molecular analysis of the different $\mathrm{scFv}$ obtained from the cell clone secreting anti-NIa monoclonal antibody but also from the original hybridoma has allowed to detect in both cases an important sequence heterogeneity which confirm the importance of the phage display technology as a complamentary approach for the classic hybridoma production by cell culture. This analysis suggested that the several scfv obtained will showed different affinities for the target protein. 